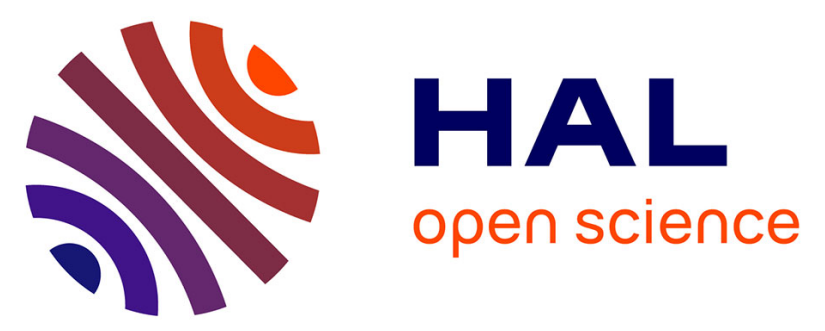

\title{
Chronologie de la déglaciation des Pyrénées françaises. Dynamique de sédimentation et contenu pollinique des paléolacs ; application à l'interprétation du retrait glaciaire
}

\author{
Valérie Andrieu-Ponel, Jacques Hubschman, Guy Jalut, Gerard Herail
}

\section{To cite this version:}

Valérie Andrieu-Ponel, Jacques Hubschman, Guy Jalut, Gerard Herail. Chronologie de la déglaciation des Pyrénées françaises. Dynamique de sédimentation et contenu pollinique des paléolacs; application à l'interprétation du retrait glaciaire. Bulletin de l'Association française pour l'étude du quaternaire, 1988, 25 (2-3), pp.55-67. 10.3406/quate.1988.1866 . hal-02735751

\section{HAL Id: hal-02735751 \\ https://hal-univ-tlse2.archives-ouvertes.fr/hal-02735751}

Submitted on 2 Jun 2020

HAL is a multi-disciplinary open access archive for the deposit and dissemination of scientific research documents, whether they are published or not. The documents may come from teaching and research institutions in France or abroad, or from public or private research centers.
L'archive ouverte pluridisciplinaire HAL, est destinée au dépôt et à la diffusion de documents scientifiques de niveau recherche, publiés ou non, émanant des établissements d'enseignement et de recherche français ou étrangers, des laboratoires publics ou privés. 


\section{per

Chronologie de la dégladation des Pyrénées françaises. Dynamique de sédimentation et contenu pollinique des paléolacs ; application à l'interprétation du retrait glaciaire

Valérie Andrieu-Ponel, Jacques Hubschman, Guy Jalut, Gérard Hérail

\section{Citer ce document / Cite this document :}

Andrieu-Ponel Valérie, Hubschman Jacques, Jalut Guy, Hérail Gérard. Chronologie de la dégladation des Pyrénées françaises. Dynamique de sédimentation et contenu pollinique des paléolacs ; application à l'interprétation du retrait glaciaire. In: Bulletin de l'Association française pour l'étude du quaternaire, vol. 25, n²-3, 1988. pp. 55-67;

doi : https://doi.org/10.3406/quate.1988.1866

https://www.persee.fr/doc/quate_0004-5500_1988_num_25_2_1866

Fichier pdf généré le 19/04/2018 


\begin{abstract}
On the basis of available modern data, it can be assumed that the beginning of the glacial retreat in the northern Pyrenees was prior to $38,4 \mathrm{KY}$. The presence of external and internal moraines in the glaciated valleys is the consequence of an irregular glacial retreat interrupted by readvanced and stagnation stages. The oldest retreat phase occurred before $38,4 \mathrm{KY}$ on the external margin of the glacial piedmont of Lourdes. In the terminal glacial basin of the Garonne, before $26 \mathrm{KY}$, a glacial recurrence (datings in process) probably contemporaneous with the building of the internal moraines is registred. Due to the lack of iterative data from other sites, we cannot say if it corresponded to a local or a more general phenomenon.

From 26-24 KY, the déglaciation was more important in the terminal glacial basins of Garonne and Ossau while in the same time the dryness increased. This climatic feature concerned the northern slope of the Pyrenees as well as the molassic piedmont, especially to the south of Toulouse where a loess deposition phase occurred which probably began before $23 \mathrm{Ky}$.

Until 16-15 KY, the glaciers retreated progressively. At middle altitude, from 16-15 KY onwards, the lacustrine or glacio fluvial deposits indicate that the mountain was widely deglaciated, the residual glaciers being only in favourable locations. In the northern slope of the Pyrenees, the déglaciation of the higher parts was virtually terminated during the dry period registred in Europe near $15 \mathrm{KY}$.
\end{abstract}

\title{
Résumé
}

Le début de la déglaciation würmienne dans les Pyrénées est antérieur à $38 \mathrm{Ka}$. La régression des glaciers a été interrompue à plusieurs reprises par des phases de progression précédées de phases de stationnement comme le montre la géométrie des édifices morainiques frontaux. La phase de retrait la plus anciennement datée a été enregistrée sur la marge glaciaire externe de Lourdes, avant $38 \mathrm{Ka}$. Dans le bassin glaciaire terminal de la Garonne, une récurrence glaciaire antérieure à $26 \mathrm{Ka}$ (datation en cours) et probablement contemporaine de la construction des moraines internes est notée. Mais, en l'absence de données itératives fournies par d'autres sites, on ne peut trancher entre l'hypothèse d'une récurrence locale et celle d'une réavancée glaciaire plus générale. A partir de 26-24 Ka débute la phase de déglaciation définitive des bassins glaciaires terminaux de la Garonne et de l'Ossau. Au même moment, la sécheresse atmosphérique s'accroît sur le versant nord des pyrénées et sur son piémont molassique, particulièrement au sud de Toulouse où des lœss sont déposés dès $24 \mathrm{Ka}$ au moins. Jusqu'à 16-15 Ka, les glaciers reculent progressivement. Les séries lacustres et fluvioglaciaires situées à moyenne altitude indiquent que la montagne est, à cette époque, largement déglacée, des glaciers résiduels pouvant demeurer dans des zones favorables. La déglaciation de la haute montagne est donc virtuellement terminée pendant la phase d'aridité maximale enregistrée en Europe vers $15 \mathrm{Ka}$. 


\title{
CHRONOLOGIE DE LA DÉGLACIATION DES PYRENÉES FRANÇAISES Dynamique de sédimentation et contenu pollinique des paléolacs : application à l'interprétation du retrait glaciaire
}

\author{
par Valérie ANDRIEU*,**, Jacques HUBSCHMAN*, Guy JALUT** \\ et Gérard HERAIL*
}

\begin{abstract}
RESUME
Le début de la déglaciation würmienne dans les Pyrénées est antérieur à $38 \mathrm{Ka}$. La régression des glaciers a été interrompue à plusieurs reprises par des phases de progression précédées de phases de stationnement comme le montre la géométrie des édifices morainiques frontaux. La phase de retrait la plus anciennement datée a été enregistrée sur la marge glaciaire externe de Lourdes, avant $38 \mathrm{Ka}$. Dans le bassin glaciaire terminal de la Garonne, une récurrence glaciaire antérieure à $26 \mathrm{Ka}$ (datation en cours) et probablement contemporaine de la construction des moraines internes est notée. Mais, en l'absence de données itératives fournies par d'autres sites, on ne peut trancher entre l'hypothèse d'une récurrence locale et celle d'une réavancée glaciaire plus générale. A rartir de 26-24 Ka débute la phase de déglaciation définitive des bassins glaciaires terminaux de la Garonne et de l'Ossau. Au même moment, la sécheresse atmosphérique s'accroît sur le versant nord des pyrénées et sur son piémont molassique, particulièrement au sud de Toulouse où des loess sont déposés dès $24 \mathrm{Ka}$ au moins. Jusqu'à $16-15 \mathrm{Ka}$, les glaciers reculent progressivement. Les séries lacustres et fluvioglaciaires situées à moyenne altitude indiquent que la montagne est, à cette époque, largement déglacée, des glaciers résiduels pouvant demeurer dans des zones favorables.La déglaciation de la haute montagne est donc virtuellement terminée pendant la phase d'aridité maximale enregistrée en Europe vers $15 \mathrm{Ka}$.
\end{abstract}

Mots-clés : Pyrénées, Chronologie, Würm, déglaciation, paléolac, sédimentologie, palynologie, géomorphologique glaciaire.

\section{ABSTRACT}

CHRONOLOGY OF DEGLACIATION IN THE FRENCH PYRENEES. Dynamic of sedimentation and pollen content of paleolakes: application to the interpretation of the glacial retreat.

On the basis of available modern data, it can be assumed that the beginning of the glacial retreat in the northern Pyrenees was prior to $38,4 \mathrm{KY}$. The presence of external and internal moraines in the glaciated valleys is the consequence of an irregular glacial retreat interrupted by readvanced and stagnation stages. The oldest retreat phase occurred before $38,4 \mathrm{KY}$ on the external margin of the glacial piedmont of Lourdes. In the terminal glacial basin of the Garonne, before $26 \mathrm{KY}$, a glacial recurrence (datings in process) probably contemporaneous with the building of the internal moraines is registred. Due to the lack of iterative data from other sites, we cannot say if it corresponded to a local or a more general phenomenon.

From 26-24 KY, the deglaciation was more important in the terminal glacial basins of Garonne and Ossau while in the same time the dryness increased. This climatic feature concerned the northern slope of the Pyrenees as well as the molassic piedmont, especially to the south of Toulouse where a loess deposition phase occurred which probably began before $23 \mathrm{Ky}$.

Until 16-15 KY, the glaciers retreated progressively. At middle altitude, from 16-15 KY onwards, the lacustrine or glaciofluvial deposits indicate that the mountain was widely deglaciated, the residual glaciers being only in favourable locations. In the northern slope of the Pyrenees, the deglaciation of the higher parts was virtually terminated during the dry period registred in Europe near $15 \mathrm{KY}$.

Key-words : Pyrenees, Chronology, Würm, deglaciation, paleolake, sedimentology, palynology, glacial geomorphology.

\footnotetext{
- CIMA UA 366 CNRS, Institut de Géographie, Université de Toulouse 2, 5, Allées A. Machado, 31058 Toulouse Cedex.

* Laboratoire de Botanique et Biogégraphie, Université de Toulouse 3, 39, Allées Jules Guesde, 31062 Toulouse Cedex, et LP 8491 CNRS, Moulis, 09200 Saint-Girons.
} 


\section{LES PYRENÉES : UNE CHAINE MONTAGNEUSE MÉRIDIONALE À L'ENGLACEMENT MODÉRÉ}

Durant le Quaternaire, les Pyrénées françaises n'ont pas connu l'ampleur des glaciations alpines en raison de leur situation latitudinale méridionale $\left(43^{\circ} \mathrm{N}\right)$, de la faible étendue de leurs bassins d'alimentation glaciaire et de l'altitude modérée de leurs reliefs. Sur le versant français, deux grands domaines glaciaires correspondant aux limites des deux grands domaines bioclimatiques actuels ressortent (fig. 1).

\section{LES FORMATIONS GLACIAIRES PYRÉNEENNES : APERÇU LITHOSTRATIGRAPHIQUE.}

Jusqu'au début des années 1970, deux interprétations chronostratigraphiques se sont opposées: d'un côté, la conception strictement monoglacialiste d'abord puis, plus nuancée, développée par $\mathrm{P}$. Barrère $(1953,1963)$, F. Taillefer $(1954,1960,1967,1969$, 1977) et G. Viers $(1960,1963,1969)$ et, de l'autre, la théorie pluriglacialiste défendue notamment par H. Alimen (1964). A partir des années 1980, les recherches pluridisciplinaires engagées sur ce thème

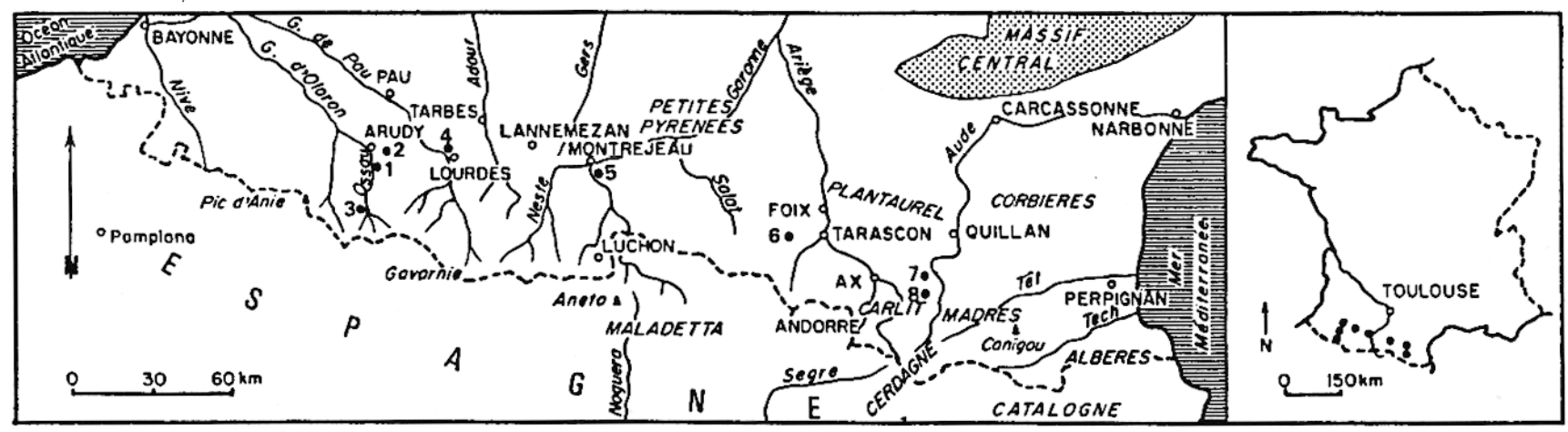

Fig. 1. - Localisation des sites glaciolacustres (GI.) et fluvioglaciaires (F1.) étudiés.

1 : Castet (Gl.), 850 m. - 2 : L'Lstarrès (GL,), 376 m. - 3 : Blous (Fl.), 1550 m. - 4 : Biscaye (Gl.), 410 m. - 5 : Barbaran (Gl.), 450 m. - 6 : Freychinède (Gl.), $1350 \mathrm{~m}$. - 7 : Ruisseau de Laurenti (Fl.), $1860 \mathrm{~m}$. - 8 : Balcère (Gl.), $1764 \mathrm{~m}$.

Fig. 1. - Location of the glaciolacustrine (GL) and glaciofturial (F) sites.

- Le domaine atlantique d'une part, qui d'ouest en est comprend les vallées du Gave d'Ossau, du Gave de Pau, de la Garonne et de l'Ariège, draînées au maximum d'emplacement par des appareils glaciaires importants dont la longueur variait entre 40 et $60 \mathrm{~km}$. Les deux plus occidentaux sont sortis de la montagne et ont étalé leur front sur le piémont entre 350 et $\mathbf{4 0 0} \mathrm{m}$ d'altitude.

- Le domaine glaciaire méditerranéen, d'autre part, concerne essentiellement les régions englacées des Pyrénées orientales. L'extension des glaciers y était fort limitée et, au maximum glaciaire, les langues émises par le massif du Carlit, le plus englacé, sont restées dans les dépressions de moyenne altitude (1 200 à $1600 \mathrm{~m}$ ) du Capcir et de la Cerdagne. ont rendu nécessaire la révision des chronologies préalablement établies. Les premiers résultats présentés par J. Hubschman en 1984 et confirmés par G. Hérail, J. Hubschman et G. Jalut en 1986 ont conduit ces auteurs à reconnaître, d'après les caractéristiques altérologiques des sédiments et les datations C 14 obtenues localement (Mardones, 1982), deux grands types de dépôts glaciaires.

- Le premier type comprend l'ensemble des moraines récentes déposées par les glaciers dans le fond des vallées et au pied des versants. Elles sont composées de sédiments peu altérés, riches en calcaire et en feldspath plagioclase et présentent des cortèges minéralogiques argileux dominés par les illites et les chlorites (Hubschman, 1984). La date de $38,4 \mathrm{Ka}(38400+2000-1200$ : Gif 5685) obtenue dans les sédiments glaciolacustres de Biscaye (Mardones, 1982), sur le piémont de Lourdes et l'identité des faciès d'altération du domaine externe et du domaine interne ${ }^{(1)}$ des vallées glaciaires pyrénéennes montrent que ces moraines relèvent d'une seule glaciation attribuable au Würm.

(1) Dans son acception purement topographique et non stratigraphique. 
- Le second ensemble correspond aux moraines anciennes présentes dans les bassins glaciaires terminaux sous la forme de placages souvent discontinus perchés sur les reliefs, ou parfois de lambeaux résiduels piégés sont des épandages fluvioglaciaires récents. Ces dépôts morainiques se caractérisent par un faciès d'altération nettement plus évolué : les sédiments, de couleur plus vive, sont dépourvus de calcaire et les fréquences des feldspaths calco-sodiques sont très faibles. Les associations argileuses se composent d'illite, de vermiculite et parfois de kaolinite (Hubschman, 1984). L'absence d'éléments datables dans ces dépôts ne permet pas de les situer avec certitude dans la chronologie, sinon de les attribuer à une période glaciaire préwürmienne.

Entre ces deux grands types de moraines s'observent, principalement dans le val d'Ariège (Andrieu, 1983), des formations de marge glaciaire caractérisées par un faciès d'altération intermédiaire entre le modèle proposé pour les moraines anciennes et celui défini dans les dépôts récents.

A la différence du modèle glaciaire classiquement reconnu dans les Alpes (Montjuvent, 1984), le dispositif morainique externe et interne du domaine glaciaire nord-pyrénéen appartient à la glaciation würmienne dont le maximum d'extension est antérieur à $38,4 \mathrm{Ka}$.

\section{LA DEGLACIATION WURMIENNE DES PYRENEES : DEFINITION ET MÉTHODES D'ÉTUDE}

La déglaciation des vallées pyrénéennes correspond à la longue période de retrait glaciaire, immédiatement postérieure à la phase d'extension glaciaire maximale et caractérisée par l'alternance de stades de progression et de phases interstadiaires. Les constructions contemporaines comprennent l'ensemble du système morainique récent et les obturations lacustres associées, déposés sur le fond des principaux axes englacés.

Des recherches pluridisciplinaires associant la géomorphologie, la sédimentologie et la palynologie ont été appliquées systématiquement à des formations glaciolacustres prélevées par sondage. La démarche stratigraphique utilisée pour l'étude des paléolacs et de leur bassin glaciaire s'inspire des principes proposés dans les années 80 par la nouvelle école de stratigraphie anglo-saxonne (Miall A.D., Reading HG., Rust B.R., Eyles N.) qui, contrairement aux pratiques stratigraphiques traditionnelles, se réfère aux modèles sédimentaires actuels pour interpréter génétiquement les unités stratigraphiques fossiles afin de définir les processus sédimentaires qui ont contrôlé leur mise en place.
L'intérêt majeur de l'étude de ces anciens lacs glaciaires est d'abord de permettre l'établissement d'une chronologie grâce aux datations obtenues par le radiocarbone ou par corrélations biostratigraphiques. Les sédiments lacustres fournissent en effet le seul matériau accessible aux datages, les moraines pyrénéennes n'ayant jusqu'à présent jamais livré de vestiges organiques datables.

En second lieu, lorsque des correspondances géodynamiques sont établies entre l'association verticale des lithofaciès lacustres et l'évolution de la géomorphologie glaciaire locale, il est possible de reconstituer la dynamique de retrait des appareils glaciaires terminaux. Lá démarche a été appliquée récemment dans la vallée de la Garonne (Andrieu, thèse en cours).

Enfin, les lacs sont d'excellents pièges à pollens dont les associations permettent de déterminer l'évolution paléophytogéographique et paléoclimatique du milieu durant la déglaciation. Des palynostratigraphies contemporaines de cette période ont été obtenues sur le piémont de Lourdes (Mardones, 1982 et Mardones et Jalut, 1983) et dans le bassin glaciaire terminal de la Garonne (Andrieu, thèse en cours) où les spectres polliniques, suffisamment riches en pollens, permettent une interprétation phytogéographique.

En raison du caractère récent des recherches entreprises et de la longueur de la démarche utilisée, la connaissance des modalités et de la chronologie de la déglaciation des principaux ensembles glaciaires pyrénéens est encore incomplète. Les données présentées dans les développements régionaux suivants correspondent donc à des résultats préliminaires.

\section{LA DÉgLACIATION DE LA VALLEE DU GAVE D'OSSAU.}

\subsection{Géomorphologie de la terminaison glaciaire du bassin d'Arudy.}

Au maximum glaciaire würmien, le glacier du Gave d'Ossau, long de $38 \mathrm{~km}$, débordait largement sur le piémont où il formait un appareil glaciaire polylobé (Taillefer, 1948, 1969; Barrère 1971; Andrieu, 1987) qui s'étalait dans le bassin d'Arudy dans quatre directions majeures. Dans l'ancienne vallée du Gave, vers Buziet (fig. 2), la principale diffluence a déposé un puissant éventail d'arcs morainiques emboîtés, large de $2 \mathrm{~km}$, et relié à la terrasse fluvioglaciaire d'Ogeu par un cône de transition caractéristique. A l'ouest d'Arudy, sur le versant qui borde le cours actuel du Gave s'observent des 


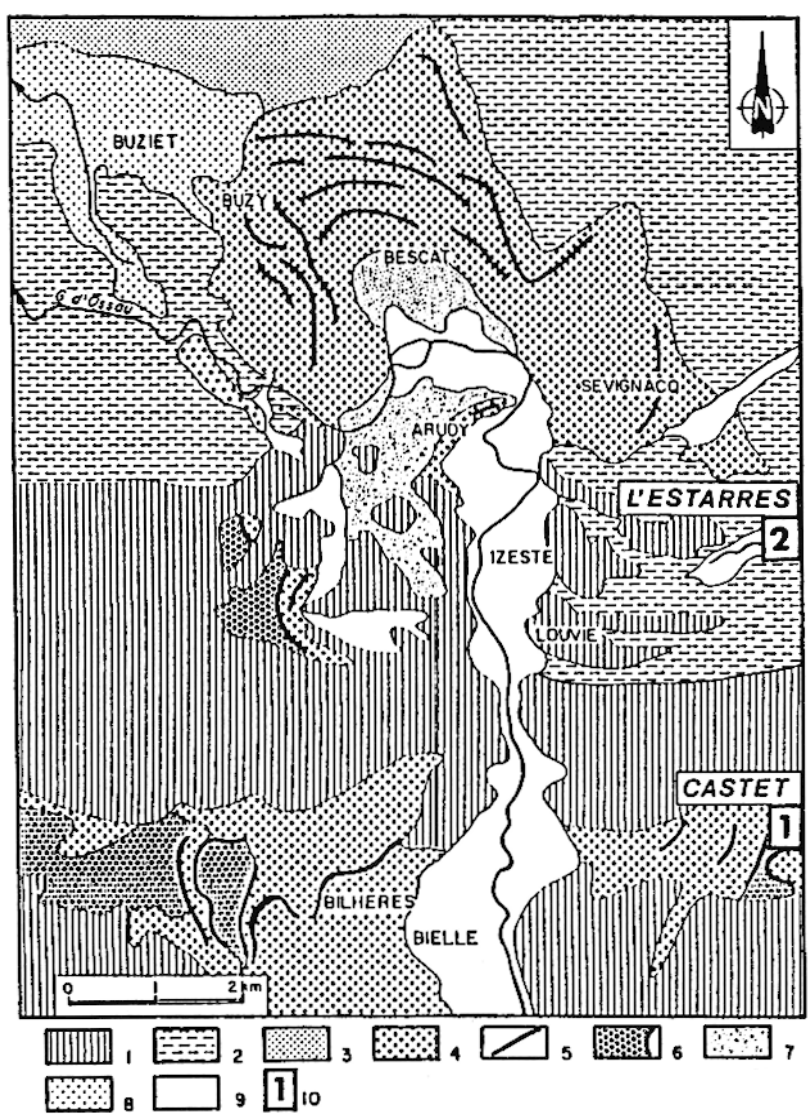

Fig. 2. - Les dépôts glaciaires de la vallée du Gave d'Ossau. 1 - Crêtes et affleurements calcaires jura-crétacés. 2 - Collines de piémont et affleurement de flyschs cénomaniens. 3 - Terrasse moyenne d'Herrère. 4 - Moraines récentes. 5 - Arcs morainiques. 6 - Plaines d'obturation fluvioglaciaire ou lacustre. 7 - Terrasse nuvioglaciaire d'Arudy. 8 - Basse terrasse d'Ogev. 9 - Terrasses et remblaiements fini-et post glaciaires. 10 - Localisation des sondages et auméro correspondant sur la figure 1.

Fig. 2. - The glacial deposits of the Gave d'Ossan nalley. 1 - Crests and bedrock of jura-cretaceous limestones. 2 - Hills of the piedmont and bedrock of cenomanian fyschs. 3 - Middle terrace of Herrère. 4 - Recent monainic deposits. 5 -Morainic ridges. 6 - Glaciofivvial or lacustrine obturations. 7 - Glaciofiuvial ternace of Arudy. 8 - Low glaciofluvial terrace of $O_{\text {gew }} 9$ - Late and postglacial deposits. 10 - Lacation of the borings and corresponding number on figure 1.

placages morainiques discontinus et, plus au sud, des obturations glaciaires fermées en aval par un cordon morainique se sont formées à l'emplacement d'anciennes dépressions karstiques. Au nord-est, la colline de Sévignacq est recouverte par une épaisse couverture morainique. Vers l'est, aucun indice géomorphologique net ne prouve la progression du glacier dans la vallée de l'Estarrès, contrairement aux observations antérieures (Barrère et Paquereau, 1960 et Paquereau et Barrère, 1964). En revanche, les sédiments glaciolacustres de l'Estarrès montrent que le lobe oriental du glacier d'Ossau a stationné sur le seuil rocheux de Louvie-Juzon (Andrieu, 1987).
L'ensemble de ces formations, synchrones de la glaciation würmienne, appartient au système des moraines externes du glaciaire d'Ossau, le domaine interne étant représenté par le petit vallum du cimetière d'Arudy (Hubschman, 1984).

Dans l'état actuel de nos connaissances, la chronologie du retrait du glacier d'Ossau est saisie de $27 \mathrm{Ka}$ à $16 \mathrm{Ka}$ grâce à l'enregistrement sédimentaire de trois séries glaciolacustres ou fluvioglaciaires (l'Estarrès, Castet et Bious) étagées entre $370 \mathrm{~m}$ et $1550 \mathrm{~m}$ d'altitude dans la vallée de l'Ossau.

\subsection{La série de l'Estarrès : 376 m (fig. 2, site no 2 )}

Dans les quatre mètres inférieurs du sondage, deux épisodes sont distingués.

- Le plus ancien, de 27 à $24,4 \mathrm{Ka}$ $(27150+-1000$ : Gif 6868 et $24400+-1000$ : Gif 6867 ), correspond à la phase de dépôt des sédiments glaciolacustres transportés par les écoulements d'eaux de fonte glaciaires libérés par le lobe oriental du glacier d'Ossau, lorsqu'il stationnait sur les reliefs dominant le bassin de l'Estarrès. Les sédiments montrent l'alternance de rythmites finement laminées et de passées limoneuses à structures massives. Ils sont caractéristiques d'une dynamique de sédimentation distale (Smith, 1978) contrôlée par des courants de densité sous-lacustres peu actifs (Shaw, 1977; Walker, 1967) et en voie de tarissement.

- Durant le second épisode, postérieur à $24,4 \mathrm{Ka}$, les effluents proglaciaires n'alimentent plus le lac et des vases calcaires limoneuses, résultant d'apports détritiques fins d'origine locale et de l'activité biologique des organismes lacustres, se déposent.

Les spectres polliniques des sédiments glaciolacustres, très pauvres en taxons et en pollen sont, de ce fait, difficilement interprétables. En revanche, les associations polliniques des dépôts limoneux, dominées par des groupements d'herbacées héliophiles à Poacées, Armoises, Chénopodiacées et Composées, traduisent le développement, dès $18,9 \mathrm{Ka}$ d'un paysage ouvert, de caractere steppique.

\subsection{La série de Castet : 850 m (fig. 2, site no 1)}

A $3 \mathrm{~km}$ en amont d'Arudy, dans une vallée affluente de l'auge glaciaire de l'Ossau, des sédiments glaciolacustres ont été prélevés dans le paléolac de Castet (Jalut et al., à paraître).

Le sondage comporte à la base plusieurs lithofaciès de diamicton (Eyles et al., 1983). Au dessus apparaissent des rythmites, à faciès proximal carac- 
téristique (Gilbert et Shaw, 1981) dont la partie supérieure est datée $25 \mathrm{Ka}(25000+-780$ : Gif 7536), suivies de dépôts d'exondation détritiques puis tourbeux.

L'association verticale des lithofaciès (Miall,1984) observés à Castet montre que vers $25 \mathrm{Ka}$ la charge sédimentaire des tributaires lacustres s'est considérablement réduite. Cette dynamique est la conséquence de l'éloignement de l'appareil glaciaire de l'Ossau et du glacier local du Jaout du littoral lacustre.

Après une phase d'abaissement progressif du lac, antérieure au Tardiglaciaire, celui-ci s'est vidangé, cessant dès lors d'enregistrer le déglaciation du bassin intramontagnard de Castet.

\subsection{La série de Bious (1550 m)}

A l'ouest du Pic du Midi d'Ossau, un sondage a été effectué à $1550 \mathrm{~m}$ d'altitude dans le remblaiement détritique de l'ombilic de Bious (fig. 1, site no 3), (Jalut et al., à paraître). Les 3 mètres inférieurs de la série, constitués d'un épais conglomérat de graviers à structures massives (lithofaciès $\mathrm{Gm}$ : Miall, 1978) recouvert d'un dépôt plus mince de limons homogènes, résultent d'apports sédimentaires fluvioglaciaires déposés pendant la phase terminale de la déglaciation de la montagne de l'Ossau. La date de $15,8 \mathrm{Ka}(15800+-240 \mathrm{Gif} 7078)$ obtenue à la base du conglomérat ( $\mathrm{Gm}-\mathrm{c} 1$ ) est synchrone de la période fixant la disparition des appareils glaciaires des vallées englacées nord-pyrénéennes vers 16-15 Ka. Toutefois, le vieillissement des autres datations de la série, antérieures à $10 \mathrm{Ka}$, et l'absence de preuves palynostratigraphiques (Pagès, 1982) liée à la stérilité pollinique de $\mathrm{Gm}-\mathrm{cl}$ posent des problèmes d'interprétation chronologique et conduisent à ne donner à cette date qu'une valeur de repère.

Les quatre cycles sédimentaires supérieurs, à grains décroissants vers le haut (lithofaciès : Gm-Sh-Fm-Cf) ont été mis en place pendant le Postglaciaire par un système fluviatile méandrant dans un milieu géomorphologique totalement déglacé.

\subsection{Les étapes de la décrue glaciaire de la vallée du Gave d'Ossau à partir de $27 \mathrm{Ka}$}

- A $27 \mathrm{Ka}$, le glacier d'Ossau est à cette époque un appareil massif qui s'étale dans le bassin d'Arudy à $400 \mathrm{~m}$ d'altitude et déborde latéralement sur les collines qui l'encadrent $150 \mathrm{~m}$ plus haut. Parallelement, la moyenne montagne connaît encore un englacement important jusque vers $25 \mathrm{Ka}$.
- Vers 25-24 Ka, les modifications des processus de dépôt observés dans les paléolacs de Castet et de l'Estarrès signalent qu'à moyenne et à basse altitude, le glacier de l'Ossau et les appareils glaciaires locaux reculent de leur position antérieure. Ce changement se produit alors que les signes d'un climat de type sec sont enregistrés au même moment dans de nombreux sites pyrénéens ou du piémont comme nous le verrons plus loin.

A $24 \mathrm{Ka}$, la déconnexion des courants de fusion glaciaire et du lac de l'Estarrès montre que les collines de Louvie-Juzon $(564 \mathrm{~m})$ ne sont plus englacées. C'est aussi probablement le cas des reliefs voisins situés à une altitude comparable.

$\mathrm{Si}$ durant l'épisode de retrait de Louvie-Juzon l'épaisseur du front glaciaire diminue considérablement, la position qu'il occupe dans le bassin d'Arudy ne peut pas être établie faute de témoins géomorphologiques ou lacustro-sédimentologiques datés. De même, les arguments qui permettraient de préciser la dynamique du retrait glaciaire font défaut.

- La décrue se poursuit encore vers $16 \mathrm{Ka}$ dans la haute vallée d'Ossau. Dès lors, la tendance particulièrement sèche du climat qui règne dans les Pyrénées à cette époque ne permet plus que l'existence de petits appareils glaciaires, dans des sites favorables.

De l'étude de la déglaciation würmienne de la vallée d'Ossau, on peut retenir un ordre de grandeur spatio-temporel : plus de 10000 ans (de 27 à $16 \mathrm{Ka}$ au moins) ont été nécessaires pour déglacer, sur une longueur de $\mathbf{4 0} \mathrm{km}$, la vallée montagnarde de l'Ossau et son piémont.

\section{LA DÉglaCIATION DE LA VALLEE DU GAVE DE PAU}

Lorsqu'il débordait sur le piémont de Lourdes au maximum glaciaire, le glacier du Gave de Pau se divisait en quatre branches, guidé dans sa progression par la disposition rayonnante des vallées préglaciaires du cône pliocène de Ger (Taillefer, 1948). Les deux principales diffluences étaient celle d'Adé, au nord et celle de Poueyferré, au nord-ouest (fig. 3). Durant la déglaciation, de nombreux arcs morainiques terminaux ont été construits dans ces deux vallées, entre 430 et $\mathbf{4 4 0} \mathrm{m}$ d'altitude.

Dans l'etat actuel de nos connaissances, les modalités du retrait glaciaire ne sont connues que sur le piémont de Lourdes dans l'axe de Poueyferré, grâce à l'étude du site glaciolacustre de Biscaye (Mardones, 1982 et Mardones et Jalut, 1983). Ce 


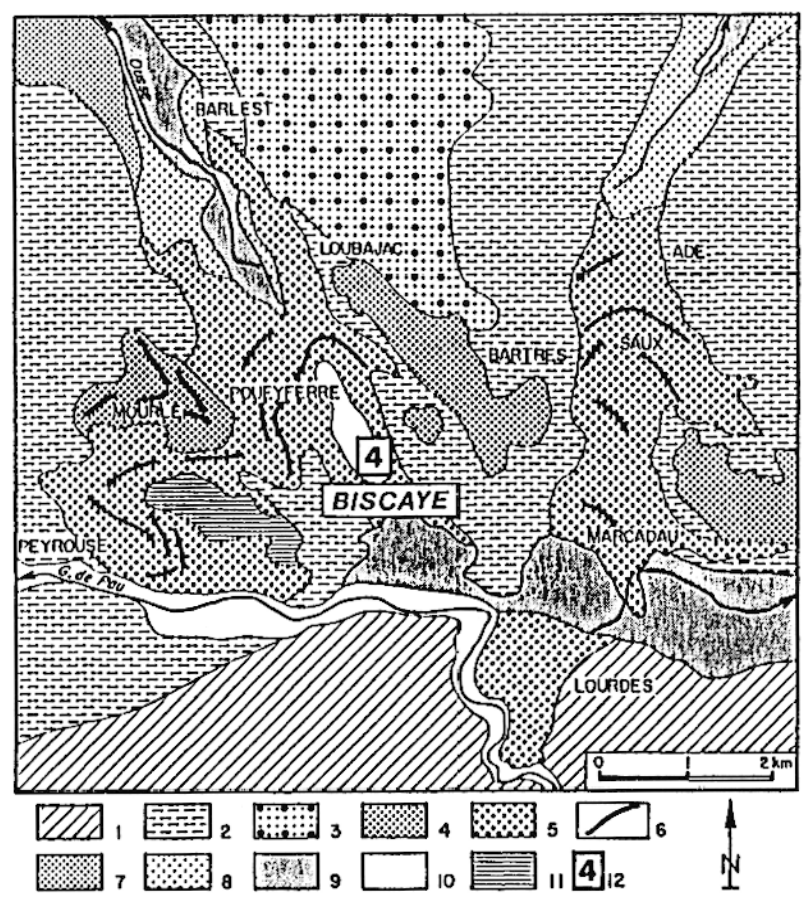

Fig. 3. - Le complexe glaciaire terminal du Gave de Pau. 1 - Montagne calcaire jura-crétacé. 2 - Collines de piémont et affleurement de nyschs cénomaniens. 3 - Cône alluvial ancien de Ger. $4^{\circ}$ - Moraines anciennes. 5 - Moraines récentes. 6 - Arcs morainiques. 7 - Terrasse moyenue d'Espoey. 8 - Basse terrasse d'Ossun. 9 - Terrasse inférieure. 10 - Lit majeur et dépression glaciolacustre de Biscaye. 11 - Lac de Lourdes. 12 - Localisation du sondage du Biscaye et uuméro correspondant sur la figure 1.

Fig. 3. - The frontal glacial complex of the Gave de Paw. 1 - Monutain of jure-cretaceous limestones. 2 - Hills of the pyreacen piedmont and bedrock of cenomanian flyschs. 3 - Old allovial fan of Ger. 4 - Old monainic deposits. 5 - Recent monainic deposits. 6- Morainic ridges. 7 - Middle terrace of Espoey. 8 - Low terrace of Ossun. 9 - Lower terrace. 10 - Flood plain and gleciolacustrine formation of Biscaye. 11 - Lake of Lowndes. 12 - Location of the boring of Biscape and corresponding number on figure 1.

paléolac s'est constitué durant l'interstade qui a succédé à la mise en place des moraines externes de Poueyferré. La date de la base du remplissage, antérieure à $38 \mathrm{Ka}(38400+2000-1200$ : Gif 5685), montre que dès cette époque, la décrue glaciaire würmienne est déjà entamée. Postérieurement, les indications sédimentologiques de Biscaye permettent de distinguer deux périodes.

- La première intervient de 38,4 à 29,5 Ka. Dans le lac de Biscaye, des sédiments glaciolacustres grossiers et homogènes puis laminés et fins se déposent. Dès $31,9 \mathrm{Ka}(31900+-2000$ : Gif 5684 ), les apports détritiques glaciaires décroissent puis disparaissent à 29,5 Ka (29500 + - 1200 : Gif 5683 ). Ces faciès caractérisent une dynamique de sédimentation régressive, liée à la déglaciation progressive du piémont de Lourdes.

- Durant la deuxième période, postérieure à 29,5 Ka, des limons calcaires et organiques d'origine locale se sédimentent. Le lac de Biscaye n'est donc plus alimenté par des courants d'eau de fonte qui, en raison du recul du glacier s'écoulent dans d'autres directions. C'est probablement dès cette époque que le Gave de Pau s'engage dans sa vallée actuelle, vers l'ouest.

S'il apparaît que, dès $29,5 \mathrm{Ka}$, le piémont de Lourdes est largement déglacé, des données manquent en revanche pour caractériser les épisodes de retraits ultérieurs et situer dans le temps, d'une part la phase de construction des moraines internes durant le stationnement du glacier dans le bassin de Lourdes, et d'autre part le moment où, libérant le bassin, il recule dans la vallée montagnarde. Pour répondre à ces questions, l'étude d'autres sites lacustres, proches du bassin glaciaire terminal de Lourdes, est envisagée.

\section{LA DEGLACIATION DE LA VALLÉE DE LA GARONNE}

Les formations glaciaires les mieux conservées et présentant la meilleure continuité géomorphologique se situent entre Barbazan et la cluse du Bazert (fig. 4). Au contraire, les sédiments glaciaires déposés par la diffluence de Tibiran-Jaunac et largement remaniés ultérieurement sont plus difficilement interprétables du point de vue géodynamique.

L'histoire du retrait du glacier garonnais sera donc perçue à travers l'évolution du lobe glaciaire du Bazert (Andrieu, thèse en cours).

\subsection{L'épisode de Burs-La Serre}

Les moraines de Burs sont des placages de bas de versant contemporains de la phase de progression glaciaire.

En revanche, l'arc frontal de La Serre (460 m) qui ferme la cluse du Bazert en aval n'est pas synchrone de cette phase de poussée mais d'une période de stationnement postérieure. En effet, cette moraine présente un profil asymétrique, inverse de celui classiquement défini pour les vallums morainiques. En outre, aucun cône de transition ne relie cette moraine à l'aval, au remblaiement alluvial de la Plaine de Rivière. L'arc de La Serre s'apparente ainsi aux moraines de retrait couramment observées en Europe du Nord (Price, 1970; Andersen et Sollid, 1971) ou dans les Alpes (Vivian, 1967) et constitue la première étape reconnue de la déglaciation de la marge glaciaire garonnaise. 


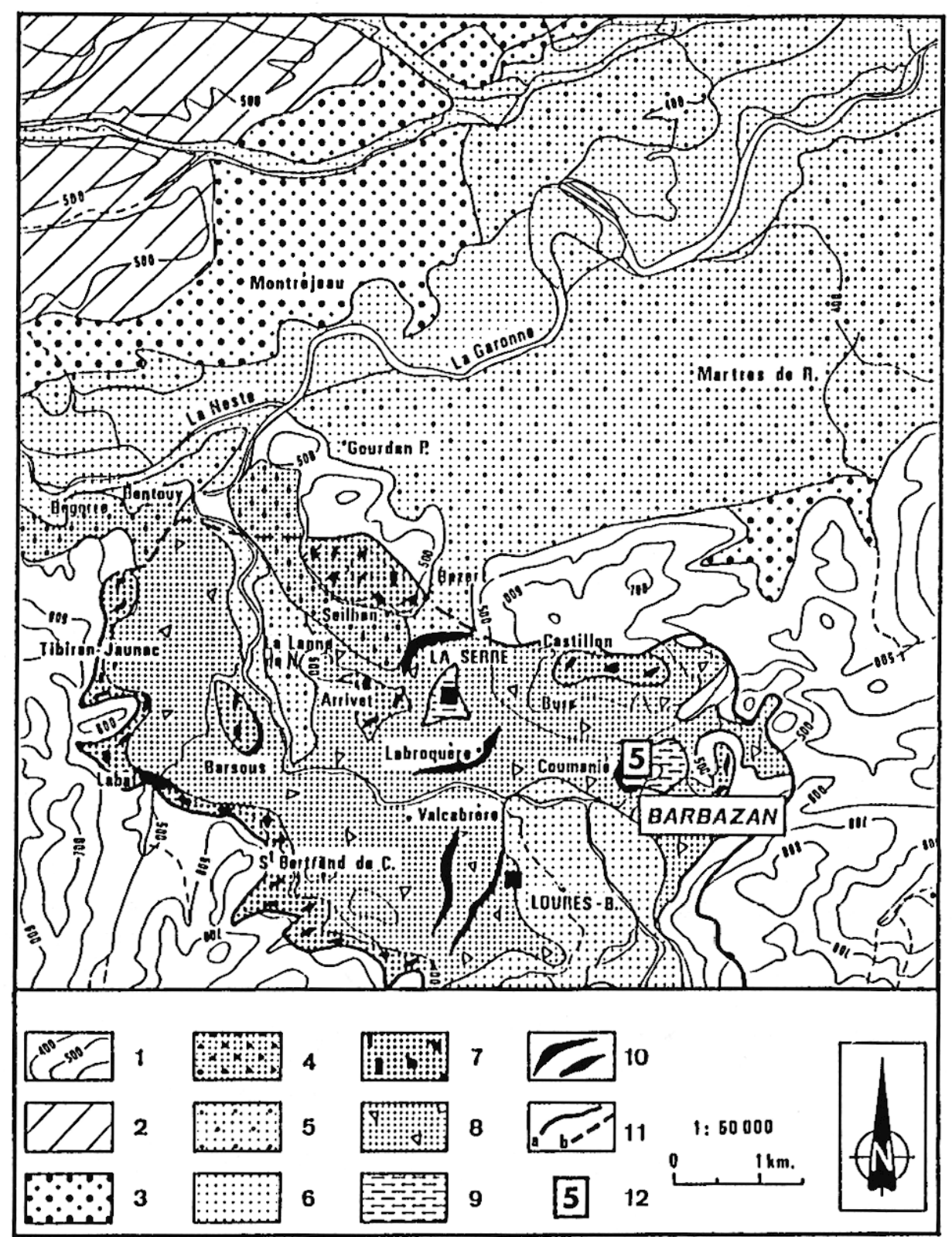

Fig. 4. - Le bassin glaciaire terminal de Barbazan - Saint-Bertrand-de-Comminges (Garoane).

1 - Reliefs dépourvus de moraine. 2 - Formatioa de Lannemezan. 3 - Moyenue terrasse de Montréjeau. 4 - Complexe des terrasses anciennes de Seilhan. 5 - Terrasse récente de La Plaine de Rivière et mappes homologues. 6 - Plaine alluviale actuelle. 7 - Moraines anciennes. 8 - Moraines et formations fluvioglaciaires récentes. 9 - Dépôts glaciolacustres. 10 - Arcs morainigues. 11 - Limites d'englacement maximum. a : limites aettes; b : limites supposées. 12 - Localisation du sondage de Barbazan et numéro correspondant sur la figure 1.

Fig. 1. - The frontal glacial basin of Barbazan - Saint-Bertrand-de-Comminges (Garonne),

1 - Bebrock withoum morainic deposits. 2 - Villafranchian finviatile formation of Lannemezan. 3 - Middle terrece of Montrejjeas. 4-Old ternaces complex of Seillhan. 5 - Recent verrace of La Plaine de Rivière and homolog formations. 6 - Recent alluvial plain. 7 - Old glacial deposits. 8 - Recent moraines and glaciofurvial deposits 9 - Glaciolacustrine deposits 10 -Monainic ridges. 11 -Maximum glacial extension limits. a : distinct limits; $b$ : probable limits. 12 - Lacation of the boring of Barbazan and corresponding number on figure 1. 


\subsection{L'interstade de La Serre}

C'est durant cet interstade que s'opère le décollement du front glaciaire garonnais des appareils morainiques externes et que se forme une obturation fluvioglaciaire entre la moraine de La Serre et le nouveau front glaciaire en amont.

Compte tenu des conditions de prélèvement (sonde de Hiller), le sondage effectué dans cette obturation ne permet pas une interprétation géodynamique correcte du remplissage fluvioglaciaire. En revanche, le résultat des premières analyses palynologiques conduisent à préciser les grands traits de la végétation régionale présente durant cette période. La palynozone SE 1 du diagramme pollinique (Andrieu, thèse en cours) définit, en effet, un paysage ouvert dominé par les Poacées, Artemisia, les Chénopodiacées, Helianthemum et les Composées. Parmi les pollens d'arbres observés, Fagus, Abies et Picea sont présents auprès du pin dominant. On note aussi la présence continue, mais dans des proportions modestes, d'essences thermophiles dont Quercus et Corylus. Ces indications palynostratigraphiques permettent aussi de situer approximativement dans le temps l'interstade de La Serre. Il semble, en effet, que cet interstade soit postérieur aux dépôts glaciolacustres de Sost 1 (Hérail et Jalut, 1986; Andrieu et Jalut 1987; Jalut, analyses en cours) synchrones de la phase de progression glaciaire würmienne et caractérisés par une végétation moins dégradée (plus riche en arbres thermophiles) que celle de La Serre. Et parallèlement, cet interstade est antérieur

à l'épisode de Barbazan - Coumanié - Labroquère comme l'indiquent à la fois sa plus grande proximité des moraines externes et les spectres polliniques de la base du sondage de Barbazan, beaucoup plus pauvres en pollens d'arbres thermophiles.

\subsection{L'épisode de Barbazan-Coumanié-Labroquère}

C'est au cours d'une ultime récurrence glaciaire que les moraines de Labroquère et celle du Coumanié ont été construites. Les premières, déposées sur les éperons rocheux du bassin de Barbazan, diffèrent génétiquement du cordon du Coumanié qui possède les caractéristiques d'une moraine sousglaciaire.

A l'est de cet édifice s'est constitué le lac proglaciaire de Barbazan. L'étude sédimentologique des $13 \mathrm{~m}$ inférieurs du sondage permet de distinguer deux périodes : l'une de progression/stationnement, l'autre de retrait glaciaire définitif.

\section{a) La période de progression/stationnement}

Elle est représentée par les quatre premiers cycles sédimentaires du remplissage lacustre durant lequel une dynamique de progression glaciaire suivie d'une phase de stationnement est progressivement enregistrée. Cette dynamique est marquée par le développement de faciès sédimentaires proximaux sur des niveaux de moins forte énergie déposés en milieu distal. L'effondrement d'une plaque de moraine sous-glaciaire dans le lac et les perturbations des rythmites subjacentes démontrent clairement la progression du glacier sur le paléolittoral lacustre. Ces événements sont peut être synchrones de la mise en place des moraines internes de Labroquère situées à moins d'1 km à l'ouest du lac de Barbazan. La date de la base du sondage, en cours d'élaboration, est probablement antérieure aux $25,6 \mathrm{Ka}$ obtenus $10 \mathrm{~m}$ plus haut.

Les spectres polliniques montrent que, durant cette période, des arbres montagnards (Hêtre, Epicéa et Sapin) se maintiennent dans des refuges voisins de l'axe englacé de la Garonne.

\section{b) La période de retrait glaciaire définitif}

Elle correspond à la dernière phase de déglaciation du bassin glaciaire terminal de la Garonne. Le début de cette phase, situé vers $26 \mathrm{Ka}$, est marqué par la déconnexion de l'appareil glaciaire et du lac de Barbazan. Durant le $5^{e}$ cycle sédimentaire, le tarissement progressif des émissaires proglaciaires se manifeste d'abord par la décroissance de l'énergie et de la compétence des courants de turbidité souslacustres responsables du dépôt des rythmites (Kuenen, 1951 a et b; Harrison, 1975; Sturm, 1979; Sturm et Matter, 1978; Gilbert et Shaw, 1981) puis par l'arrêt de la sédimentation turbiditique. La formation des rythmites ne résulte plus, dès lors, que de processus de décantation intralacustre.

Les spectres polliniques signalent dès $25,6 \mathrm{Ka}$ (25600+ - 800: Gif 7579$)$ le développement de formations herbacées essentiellement constituées de Poacées, d'Armoises et de Chénopodiacées auxquelles sont associées divers autres taxons héliophiles tels les Composées tubuliflores et liguliflores, $\mathrm{He}$ lianthemum et Ephedra. La composition des ces groupements végétaux et les aires de répartition actuelles de certains de ces taxons, notamment les Armoises et les Chénopodiacées, permettent de penser que l'on est en présence d'un couvert végétal ouvert dont la physionomie est essentiellement conditionnée par la faiblesse des précipitations. $\mathrm{Ce}$ facteur climatique est probablement à l'origine du recul du Hêtre, connu pour ses exigences en matière d'humidité et de nébulosité.

L'évolution de la végétation dès $25,6 \mathrm{Ka}$ n'est pas uniquement due à un processus naturel de coloni- 
sation des milieux libres de glace. Elle est principalement la conséquence d'un climat de type sec qui provoque, au même moment, la décrue définitive des appareils glaciaires de la Garonne et de l'Ossau (vers 25-24 Ka, voir plus haut) en raison du déficit des réserves neigeuses des bassins d'altitude. L'accroissement de la sécheresse atmosphérique est enfin corroboré par le dépôt de loess sur le terrefort toulousain, à $100 \mathrm{~km}$ au nord du front montagneux des Pyrénées, dès $23 \mathrm{Ka}$ au moins (Revel et Bourgeat, 1981). En Europe centrale, c'est à des dates proches $(26,7-25 \mathrm{Ka})$ qu'est signalé le début de la phase de dépôt maximal des loess (Kukla et Koci, 1972). De tels processus d'éolisation nécessitent un climat sec caractérisé par un déficit hydrique atmosphérique important. A l'époque actuelle, la déflation éolienne est un phénomène couramment observé sur les marges des sandurs proglaciaires de l'slande ou du Spitzberg, colonisées par une végétation steppique clairsemée (Embelton et King, 1968; Tricart et Cailleux, 1967).

Ainsi, la convergence des processus phytogéographiques, glaciaires et éoliens n'est pas fortuite. Elle relève d'une dynamique globale contrôlée par le climat.

A partir d'une date antérieure à $15,8 \mathrm{Ka}$ $(15800+-270$ : Gif 7623$)$, (datation en cours), dans le lac de Barbazan, le dépôt de vases calcaires silteuses sur les sédiments glaciolacustres montre qu'une dynamique de sédimentation détritique et biogénique locale prévaut. Ce changement de faciès prouve aussi que les émissaires proglaciaires ne parviennent plus jusqu'au littoral lacustre en raison de la réorganisation des écoulements fluviatiles dans les zones distales du front glaciaire, comme cela s'observe fréquemment dans les plaines alluviales des régions récemment déglacées (Jopling et Mc Donald, 1975; Boothroyd et Nummedal, 1978). Parallèlement, les processus de steppisation du paysage s'accentuent et la sécheresse climatique s'accroît.

Ces événements sont synchrones de la période d'aridité maximale repérée entre 16 et $15 \mathrm{Ka}$ dans l'ensemble de l'Europe nord-occidentale (Van Campo, 1985). A cette époque, le glacier de la Garonne reste confiné à la haute vallée montagnarde.

\subsection{La déglaciation de la vallée de la Garonne : esquisse chronologique et paléoenvironnementale}

La déglaciation de la marge glaciaire garonnaise commence après le stade de dépôt des moraines terminales de Burs, contemporain du maximum d'englacement pyrénéen. L'interstade de La Serre est antérieur à celui qui permet la formation du lac proglaciaire de Barbazan. Dans ce site, les sédiments ont enregistré les signes d'une récurrence glaciaire, probablement synchrone de la construction des moraines internes de Valcabrère. Durant cette période, les spectres polliniques de Barbazan traduisent le maintien de refuges à Pins, Hêtres, Epicéas et Sapins.

Le début du retrait glaciaire définitif du bassin de Barbazan-St Bertrand de Comminges se produit vers $26 \mathrm{Ka}$ et parallèlement, un climat de type sec se développe dans les Pyrénées. A cette date, le front glaciaire se situe encore à proximité des moraines internes. Postérieurement, le glacier a évolué dans la vallée montagnarde de la Garonne.

\section{LA DÉGLACIATION DU VAL D'ARIÈGE}

Contrairement aux complexes terminaux précédents, les formations glaciaires ariégeoises sont dispersées entre le bassin de Tarascon et la cluse de Foix (fig. 5) que l'on considère le plus souvent comme le point d'avancée maximale du glacier de l'Ariège (Taillefer, 1985, 1987).

A l'ouest de Foix, l'obturation glaciolacustre de l'Arget (Taillefer et Durand, 1959) témoigne d'une phase de stationnement, peut être postérieure au maximum glaciaire. En amont, dans la vallée du Sios, le cône détritique de St Paul de Jarrat résulte de la progradation d'une branche diffluente du glacier ariégeois sur des formations deltaïques de marge glaciaire (Andrieu, 1983). Compte tenu du degré d'altération des sédiments, la récurrence du Sios est assurément plus ancienne que les épisodes correspondant au dépôt des moraines de Garrabet, Arignac et La Bernière, dans la gorge de Mercus. La moraine de Garrabet est contituée de trois ondulations soudées auxquelles se relie la terrasse fluvioglaciaire de Montgaillard. Trois kilomètres en amont, le complexe morainique d'Arignac, caractérisé par la progradation de dépôts morainiques sur des sédiments deltaïques, et le cordon de La Bernière correspondent à deux pulsations glaciaires. Aucune datation ne permet de situer chronologiquement ces événements. Les faciès d'altération peu évolués des matrices morainiques soulignent cependant leur caractère récent (Andrieu, 1983).

Les seuls repères chronologiques dont on dispose dans la vallée de l'Ariège proviennent des datations $\mathrm{U} / \mathrm{Th}$ effectuées sur les planchers calcitiques du remplissage karstique de Niaux-Lombrives-Sabart, dans le bassin de Tarascon. D'après Sorriaux (1982) et Bakalowitcz et al. (1984), la glaciation würmienne 


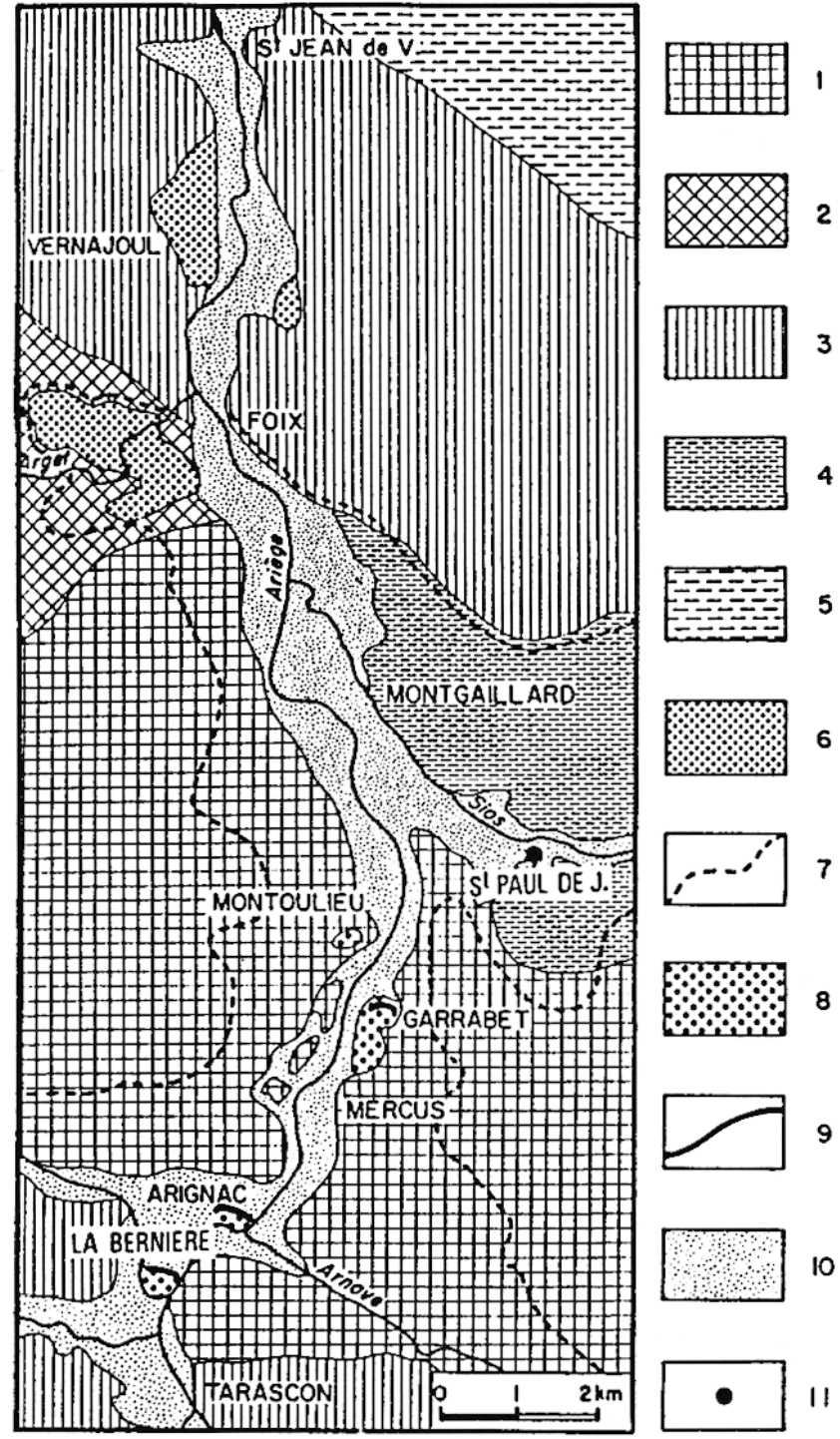

Fig. 5. - Les dépôts glaciaires du val d'Ariège.

1 - Massif paléozoìque de l'Arget-Arize. 2 - Granite de la Barguillère. 3 - Crêtes calcaires. 4 - Flyschs et marmes du bassin de Foix-Montgaillard. 5 - Collines molassiques. 6 - Dépôts glaciaires et fluvioglaciaires anciens. 7 - Limites d'englacement maximum supposées. 8 - Dépôts glaciaires récents. 9 - Arcs morainiques. 10 - Terrasses fluvioglaciaires récentes. 11 - Cône détritique de Saint-Paul-de-Jarrat.

Fig. 5. - The glacial deposits of the Ariège Valley.

1. Paleozoic massif of Arget-Arize. 2 - Granite of Barguillere. 3 - Calcareous crests. 4 - Flyschs and marts of the basin of Foix-Montgaillard. 5 - Molassic hills. 6 - Old glacial and glaciofluvial deposits. 7 - Probable limits of the maximum glacial extension. 8. Recent glacial deposits. 9-Morainic ridges. 10 - Recent glaciofluvial terraces. 11 - Outwash fan of Saint-Paul-de-Jarrat.

débute vers $90 \mathrm{Ka}$ et est pratiquement achevée vers $20 \mathrm{Ka}$.

A moyenne altitude, dans le bassin de Vicdessos, le remplissage glaciolacustre de Freychinède (1 $350 \mathrm{~m}$ ) montre qu'à partir de $21 \mathrm{Ka}$ $(21300+-760$ : Gif 4957$)$, le glacier local des Trois-Seigneurs régresse (Jalut et al., 1982; Jalut et al., à paraître). Ses eaux de fonte cessent d'alimenter le paléolac dès $14,7 \mathrm{Ka}(14700+-800$ : Gif 5018 ), en raison du recul du front glaciaire. L'évolution paléoécologique ultérieure, caractérisée par une phase de colonisation arbustive et arborescente à Genévriers et Bouleaux, permet de situer vers $14 \mathrm{Ka}$ la déglaciation totale de la haute montagne ariégeoise.

\section{LA DEGLACIATION DES PYRÉNEES MÉDITERRANÉENNES}

Le dispositif morainique le plus important des Pyrénées méditerranéennes a été construit sur le versant sud-est du massif du Carlit (2921 m) par une série de petites langues glaciaires dont la plus longue était celle de la Têt $(16 \mathrm{~km})$. En amont de MontLouis, vers $1600 \mathrm{~m}$ d'altitude, cet appareil glaciaire a édifié un vaste " amphithéatre morainique " dont le vallum externe atteint plus de $100 \mathrm{~m}$ de haut (Viers, 1961, 1963; Taillefer, 1969).

Les rares datations dont nous disposons donnent des âges postérieurs à $15 \mathrm{Ka}$. Elles sont tirées de l'étude palynologique de deux sites d'altitude : le gisement de Balcère, $1764 \mathrm{~m}$ (fig. 1, site n० 8), (Van Campo et Jalut, 1969), et celui du Ruisseau du Laurenti, 1860 m (fig. 1, site no 7), (Jalut, 1974), De la réinterprétation actualisée des palynostratigraphies, il ressort que, dans le haut Capcir, le glacier qui occupait le vallon de Balcère s'est rétracté au dessus de $1800 \mathrm{~m}$ dès $15 \mathrm{Ka}$. Dans le Quérigut, après 14,5-14 Ka, le glacier du Laurenti ne pouvait être situé qu'à une altitude supérieure à $1900 \mathrm{~m}$, dans le massif du Roc Blanc (2 $498 \mathrm{~m}$ ).

Dans les Pyrénées méditerranéennes, le manque de repères chronologiques ne permet pas de reconstituer objectivement la déglaciation des massifs englacés. Une campagne de sondage, visant à éclairer ce problème, est en cours de réalisation.

\section{SYNTHÈSE PROVISOIRE \\ DES CONNAISSANCES ACTUELLES DE LA DEGLACIATION WURMIENNE DES PYRÉNEES FRANÇAISES}

Dans les Pyrénées françaises, la décrue glaciaire débute après la phase d'englacement maximal, antérieure à $38 \mathrm{Ka}$. Sur l'ensemble du versant nord de la Chaîne, la géométrie complexe du dispositif morainique récent de la marge glaciaire frontale, est la conséquence d'une dynamique de retrait irrégulière marquée par la succession de stades de progres- 
sion/stationnement et de phases de régression. Régionalement, la plus ancienne phase de retrait datée se produit sur le piémont glaciaire de Lourdes, avant $38 \mathrm{Ka}$.

A une date antérieure à $26 \mathrm{Ka}$, dans le bassin glaciaire terminal de la Garonne, une récurrence glaciaire (datation en cours) probablement synchrone du dépôt des moraines internes, est enregistrée. Faute de données itératives recueillies jusqu'à présent en d'autres sites, aucun argument ne permet pour l'heure de savoir si cette récurrence est locale et, dans ce cas, particulière au glacier de la Garonne, ou si cette dynamique concerne l'ensemble des glaciers nord-pyrénéens.

A partir de 26-24 $\mathrm{Ka}$, les processus de déglaciation s'intensifient dans les bassins glaciaires terminaux de la Garonne et de l'Ossau en même temps que s'accroît la sécheresse atmosphérique. Cette tendance climatique concerne les Pyrénées et son piémont molassique, notamment au sud de Toulouse où une intense période d'éolisation est enregistrée dès $23 \mathrm{Ka}$ au moins.

Jusqu'à 16-15 Ka, le recul des glaciers s'effectue progressivement dans les vallées montagnardes. A ces dates, les séries sédimentaires d'altitude de l'Ossau, l'Ariège et les Pyrénées méditerranéennes montrent que la haute montagne pyrénéenne est largement déglacée. Seuls des glaciers relictuels peuvent subsister dans des sites favorables. La déglaciation de la haute montagne pyrénéenne est donc pratiquement achevée lors de la période d'aridité maximale que connaît l'ensemble de l'Europe du nord-ouest vers $15 \mathrm{Ka}$.

\section{BIBLIOGRAPHIE}

Alimen H., 1964. - Le quaternaire des Pyrénées de la Bigorre. Mémoires du Service de la Carte Géologique de France, Paris, 286 pp.

Andersen J.L. et Sollid J.L., 1971. - Glacial chronology and glacial geomorphology in the marginal zones of the glaciers, Midtdalssbreen and Nigardsbreen, south Norway. Norsk Geogr. Tidsskr, 25,1, p. $1-30$.

Andrieu V., 1983. - Les coupes ariégeoises de St Paul de Jarrat et Bompas-Arignac. Analyse sédimentologique et signification morphologique. Mémoire de Maîtrise, Université de Toulouse-le Mirail, 304 pp.

Andrieu V., 1987. - Le paléoenvironnement du piémont nord-pyrénéen de $27000 \mathrm{BP}$ au Postglaciaire : la séquence de l'Estarrès (Pyrénées atlan- tiques, France) dans le bassin glaciaire d'Arudy. C.R. Acad. Sc. Paris, t. 304, II, 2, p. 103-108.

Andrieu V. et Jalut G., 1987. - Le paléoenvironnement de la moyenne montagne garonnaise de la phase de progression du glacier würmien à l'actuel. Approche palynologique et sédimentologique préliminaire. 10e Symposium A.P.L.F. : "Palynologie, Ecologie, Paléoécologie ", Talence, 1987, Abstracts, CEGET-CNRS Ed., p. 17-18.

Bakalowicz M., Sorriaux P., Ford D.C., 1984. Quaternary glacial events in the Pyrenees from $U$ series dating of speleothems in the Niaux-Lombrives-Sabart caves, Ariege, France. Norsk. Geogr., Tidsskr., 38, p. 193-197.

Barrère P., 1953. - Equilibre glaciaire actuel et quaternaire dans l'ouest des Pyrénées centrales. Rev. géogr. Pyr. sud-ouest, 24, (2), p. 116-134.

Barrère P., 1963. - La période glaciaire dans l'ouest des Pyrénées centrales franco-espagnoles. Bulletin de la Société Géologique de France, 7, (5), p. 516-526.

Barrère P., 1971. - Le complexe morainique d'Arudy. Photo Interprétation $\mathrm{n}^{\circ} 3$, fasc. 3, p. 19-25.

Barrère P. et Paquereau M., 1960. - Les tourbières bombées de la vallée de l'Estarrès et leurs enseignements morphologiques. Rev. Géogr. Pyr. Sud-Ouest, 31, (2), p. 165-180.

Boothroyd J.C. et Nummedal D., 1978. - Proglacial braided outwash : a model for humid alluvial-fan deposits, in Miall A.D. (ed), Fluvial Sedimentology. Mem. Can. soc. Petrol Geol., 5, p. 641-668.

Embelton C. et King C.A.M., 1968. - Glacial and periglacial geomorphology, Arnold, Londres, $608 \mathrm{p}$.

Eyles N., Eyles C.H. Day T.C., 1983. - Sedimentologic and palaeomagnetic characteristics of glaciolacustrine diamict assemblages at Scarborough Bluffs, Ontario, Canada, in Evenson E.B., Schluchter Ch., Rabassa J. (ed), Tills and related deposits. A.A. Balkema, p. 23-45.

Gilbert R. et Shaw J., 1981. - Sedimentation in proglacial Sunwapta Lake, Alberta. Can J. Earth Sci, 18, p. 81-93.

Harrisson S.S., 1975. - Turbidite origin of glaciolacustrine sediments, Woodcock lake, Pennsylvania. Journal of Sedimentary Petrology, 45, 3, p. 738-744.

Hérail G. et Jalut G., 1986. - L'obturation de Sost (Haute-Garonne) : données nouvelles sur le paléoenvironnement de la phase de progression du glacier würmien dans les Pyrénées centrales. C.R. Acad. S. Paris, t. 303, II, 8, p. 743-747.

Hérail G., Hubschman J., Jalut G., 1987. - Quaternary glaciation in the French Pyrenees, in Sibrava Y., Bowen D.Q., Richmond G.M. (ed.) : 
Quaternary glaciations in the northern hemisphere. Quaternary Science Reviews, Pergamon, 5, p. 397-402.

Hubschman J., 1984. - Glaciaire ancien et glaciaire récent: analyse comparée de l'altération de moraines terminales nord-pyrénéennes. Rev. Géogr. Pyr. Sud-Ouest, no spéc. : "Montagnes et Piémonts », p. 313-331.

Jalut G., 1974. - Evolution de la végétation et variations climatiques durant les quinze derniers millénaires dans l'extrémité orientale des Pyrénées. Thèse, Université de Toulouse III, 181 pp.

Jalut G., Delibrias G., Dagnac J., Mardones M., Bouhours M., 1982. - A palaeoecological approach to the last 21000 years in the Pyrenees : the peat-bog of Freychinède (alt. : $1350 \mathrm{~m}$, Ariège, South France). Palaeogeogr., Palaeoclimatol., Palaeoecol., 40, p. 321-359.

Jalut G., Andrieu V., Delibrias G., Pagès P., (à paraître). - Paleoenvironnement of the valley of Ossau (French Western Pyrenees) during the last 27000 years.

Jalut G., Andrieu V., (à paraître). - Syntheses for type regions Fzg, Fn2 and Fr in France: the Northern slope of the Pyrenees. I.G.C.P. Project $158 \mathrm{~b}$. B. Berglund ed.

Jopling A.V., Mc Donald B.C., 1975. - Glaciofluvial and glaciolacustrine sedimentation. Society of economic paleontologists and mineralogists, Spec. Pub., no 23, 320 pp.

Kuenen Ph. H., 1951a. - Turbidity currents as the cause of glacial varves. Journal of Geology, 59, p. 507-508.

Kuenen Ph. H., 1951b. - Mechanics of varve formation and the action of turbidity currents. Geol. Fören. Förhandl., Bd 73, H.1, p. 69-84.

Kukla G.J., Koci A., 1972. - End of the last interglacial in the loess record. Quatern. Res., 2, p. 374-383.

Mardones M., 1982. - Le Pléistocène supérieur et l'Holocène du piémont de Lourdes : le gisement de Biscaye. Thèse, Université de Toulouse-le Mirail, 96 pp.

Mardones M., Jalut G., 1983. - La tourbière de Biscaye (alt. : $409 \mathrm{~m}$, Hautes-Pyrénées) : approche paléoécologique des $\mathbf{4 5} \mathbf{0 0 0}$ dernières années. Pollen et Spores, 25, (2), p. 163-212.

Miall A.D., 1984. - Principles of sedimentary basin analysis. Springer-Verlag, Berlin, 490 pp.

Montjuvent G. (éd.), 1984. - Quaternaire. Synthèse Géologique du Sud-Est de la France. Mémoire du B.R.G.M., no 125, p. 521-580.
Pagès P., 1982. - Histoire de la végétation en Haute Vallée d'Ossau. Mémoire de D.E.A., Université de Toulouse III, 45 pp.

Paquereau M., Barrère P., 1964. - Palynologie et morphologie quaternaire dans les environs d'Arudy. Actes du 4 e Congrès International d'Etudes Pyrénées, Pau-Lourdes, t. 4, Section IV, p. 18-25.

Price R.J. 1970. - Moraines at Fjallsjökull, Iceland. Arctic and Alpine Research, 2, p. 27-42.

Revel J.C., Bourgeat F., 1981. - Sols fossiles du terrefort toulousain et leur signification climatique. Bull de l'A.F.E.Q., 7-8, (3-4), p. 149-158.

Shaw J., 1977. - Sedimentation in an alpine lake during deglaciation, Okanagan valley, British Columbia, Canada. Geografiska Annaler, vol. 59, no 3-4, p. 221-240.

Smith N.D., 1978. - Sedimentation processes and patterns in a glacier-fed lake with low sediment imput. Can. J. Earth Sci., 15, p. 741-756.

Sorriaux P., 1982. - Etude sédimentologique des remplissages du système karstique de Niaux-Lombrives-Sabart. Thèse, Université de Toulouse III, 225 p.

Sturm M., 1979. - Origin and composition of clastic varves, in Schluchter Ch. (ed): "Moraines and varves: origin, genesis, classification ", Balkema A.A. (Pub.), Rotterdam, p. 281-285.

Sturm M., Matter A., 1978. - Turbidites and varves in lake Brienz (Switzerland) : depositing of clastic detritus by density currents. Spec. Publs int. Ass. Sediment., 2, p. 147-168.

Taillefer F., 1948. - Les bassins glaciaires d'Arudy et de Lourdes et le détournement des Gaves d'Ossau et de Pau. Essai de morphologie comparée. Mélanges géographiques D. Faucher, Toulouse, t. 1, p. 449-465.

Taillefer F., 1954. - La terminaison du glacier de la Garonne au sud de Montréjeau. Bull. Sect. Géogr. Com. Trav. Hist. et Scient., 66, p. 271-282.

Taillefer F., 1960. - Les terrasses d'obturation glaciaire de la vallée de Saurat (Ariège). Rev. Géogr. Pyr. Sud-Ouest, 31, 1, p. 45-61.

Taillefer F., 1967. - Extend of Pleistocene glaciation in the Pyrenees, in Wright H.F. and Osborne W.D. (eds) : "Arctic and alpine environments", p. 255-266, Indiana Univ. Press.

Taillefer F., 1969. - Les glaciations des Pyrénées. Bull de l'A.F.E.Q., Supp. 8e Cong. I.N.Q.U.A., Paris, p. 19-32.

Taillefer F., 1977. - Le glacier de l'Ariège dans le bassin de Tarascon. Rev. Géogr. Pyr. Sud-Ouest, 48, (3), p. 269-286. 
Taillefer F., 1985. - Idées actuelles sur les glaciations dans les Pyrénées de l'Ariège. Rev. Géogr. Pyr. Sud-Ouest, 56, (3), p. 323-338.

Taillefer F., 1987. - Les glaciations dans les Pyrénées ariégeoises. Bulletin de la Société Ariégeoise des Sciences, Lettres et Arts, p. 35-54.

Taillefer F. et Durand M.C., 1959. - Les argiles à varves de Sibian près de Foix. Bulletin de la Société d'Histoire naturelle de Toulouse, 94, p. 254-261.

Tricart J. et Cailleux A., 1967. - Le modelé des régions périglaciaires, Sedes, Paris, 512 pp.

Van Campo M., 1985. - Relations entre la végétation de l'Europe et les températures de surfaces océaniques après le dernier maximum glaciaire Pollen et Spores, 26, 3-4, p. 497-518.

Van Campo M. et Jalut G., 1969. - Analyse pollinique de sédiments des Pyrénées orientales : le lac de Balcère (1754 m). Pollen et Spores, 11, 1, p. 116-126.

Viers G., 1960. - Pays basque français et Baretous. Le relief des Pyrénées occidentales et leur piémont. Privat, Toulouse, 604 pp.
Viers G., 1961. - Le glaciaire du massif du Carlit (Pyrénées orientales). Rev. Géogr. .Pyr. Sud-Ouest, 32, p. 5-33.

Viers G., 1963. - Les moraines externes de la Cerdagne et du Capcir (Pyrénées orientales, France) et leurs rapports avec les terrasses alluviales. 6e Int. Cong. Quaternary, Warsaw, 1961, Lodz III, p. 385-393.

Viers G., 1969. - Cerdagne et Capcir. Livret-guide excursion A-6, Pyrénées orientales, centrales, Rousillon, Languedoc occidental. $\sigma^{e}$ Congr. I.N.Q.U.A., Paris, p. 67-72.

Vivian R., 1967. - Morphologie des marges proglaciaires : sur quelques aspects caractéristiques de l'écoulement des eaux et de ses effets morphologiques dans les marges proglaciaires alpines. Revue de Géographie Alpine, 55, 2, p. 267-289.

Walker R.G., 1967. - Turbidite sedimentary structures and their relationships to proximal and distal depositional environments. Journal of Sedimentary Petrology, 37, 1. p. 25-43. 